\title{
A Nonlinear Adaptive Controller for Airborne Wind Energy Systems
}

\author{
Sanket Diwale ${ }^{1}$, Andrea Alessandretti ${ }^{12}$, Ioannis Lymperopoulos ${ }^{1}$ and Colin N. Jones ${ }^{1}$
}

\begin{abstract}
A nonlinear adaptive path following controller for a kite based airborne wind energy system is presented. For a given desired geometric path, we provide necessary conditions for closed-loop convergence of the kite to a tube centered around the desired path. The proposed controller is adapts for the case of unknown wind vector and kite parameters. The effectiveness of the approach is demonstrated via numerical simulations for multiple desired shapes of the geometric path and for varying desired tether length references.
\end{abstract}

\section{INTRODUCTION}

A common operating mode for kite based Airborne Wind Energy (AWE) systems is the "pumping cycle". This operation requires the kite to reel out at a desired rate as it flies a high energy extraction manoeuvre, then reel back in with a low energy consumption manoeuvre to produce a net positive energy generation cycle (see, e.g., [1], [2]).

While the desired trajectory for the vehicle can be precomputed using numerical optimal control solvers ([3], [4], [5]), the motion control of the system presents numerous challenges due to the nonholonomic properties of the system and the limited control inputs available. In fact, since the main driving force is provided by the wind, the vehicle can only follow time-profiles along the reference trajectory that are coherent with the wind. Due to this reason, most of the control schemes as explored in [6],[7],[8],[9], focus on tracking motion of the vehicle in a plane perpendicular to the tether and control the tether length in a decoupled fashion. This alone, cannot guarantee closed loop bounded tracking of an optimal/desired trajectory. Nonlinear MPC schemes for trajectory tracking have also been explored in literature (e.g., [10], [11]) which require real-time estimation of wind speed and vehicle parameters.

Motivated by these observations, we propose a pathfollowing controller, where the reference is not a timeparametrised trajectory but rather a geometric trajectory parametrised on a generic path parameter. Such a parameter is then driven by the controller resulting in feasible trajectories coherent with the wind field. The controller is also extended to the case of unknown wind velocity vector and vehicle parameters. In both cases, we provide necessary conditions for convergence of the vehicle position to an arbitrary small neighbourhood of the desired trajectory.

The organization of the paper is as follows. In Section II a kinematic model for the kite is introduced. Section III discusses the main result, controller design, for the nominal

This work is funded by the Sinergia project "Autonomous Airborne Wind Energy" $\left(\mathrm{A}^{2} \mathrm{WE}\right)$ of the Swiss National Science Foundation (SNSF).

1 École Polytech. Fédérale de Lausanne (EPFL), Lausanne, Switzerland.

2 Institute for Systems and Robotics (ISR), Instituto Superior Tecnico (IST), Lisbon, Portugal.

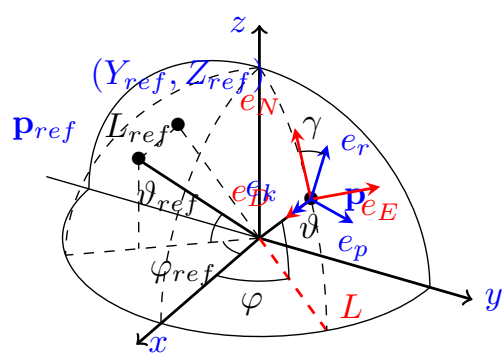

Fig. 1: Coordinate frames, Kite and Reference Path States

and adaptive cases. Section IV gives the results from the numerical tests of the controller. Section V summarizes the results described in the paper. We collect in the Appendix some of the exact expressions and background computation required for the controller.

\section{Kite ModeL}

\section{A. Coordinate Frames}

We use coordinate frames consistent with those used in [9]. We refer to Fig. 1 for a graphical illustration.

An inertial frame $\{G\}$ is attached to the ground, with basis vectors $(x, y, z)$, and a moving frame $\{K\}$ is attached to the body of the kite, with basis vectors $\left(e_{r}, e_{p}, e_{k}\right)$. Let $\mathbf{p}$ denote the position of the origin of the kite frame written in the ground frame, and let $(L, \vartheta, \varphi)$ denote its polar coordinate representation. Here, $L$ represents the tether length and $\vartheta$ and $\varphi$ denote the elevation and azimuth angle, respectively.

For any fixed tether length the kite moves on a sphere. We consider an intermediate right handed coordinate frame $\{N\}$ centered in $\mathbf{p}$ with basis vectors $\left(e_{N}, e_{E}, e_{D}\right)$, with $e_{N}$ pointing in the direction of the sphere's apex and $e_{D}$ pointing towards the sphere's center. Using this intermediate frame, and assuming always non-zero velocity, we denote by $\gamma$ the angle that the kite's velocity vector projected on the $e_{N}-e_{E}$ plane, tangent to the sphere, forms with $e_{N}$. Then, the kite frame $\{K\}$ is obtained by rotating the frame $\{N\}$ about $e_{D}$ by the angle $\gamma$.

We denote by $R_{G N}, R_{N K}$ the rotation transformation matrices from the ground frame $\{G\}$ to local north frame $\{N\}$, and from the local north $\{N\}$ to the kite's body fixed frame $\{K\}$, respectively, i.e.,

$$
\begin{aligned}
R_{G N} & =\left(\begin{array}{ccc}
-\sin \theta \cos \varphi & -\sin \varphi & -\cos \vartheta \cos \varphi \\
-\sin \vartheta \sin \varphi & \cos \varphi & -\cos \vartheta \sin \varphi \\
\cos \vartheta & 0 & -\sin \vartheta
\end{array}\right) \\
R_{N K} & =\left(\begin{array}{cc}
\bar{R}_{N K} & \mathbf{0} \\
\mathbf{0} & 1
\end{array}\right), \bar{R}_{N K}=\left(\begin{array}{cc}
\cos \gamma & -\sin \gamma \\
\sin \gamma & \cos \gamma
\end{array}\right) .
\end{aligned}
$$




\section{B. Kinematic Model}

Similar to the model used in [7], but extended to a variable line length setting and expressed in different coordinate frames, the kinematic model of the kite can be written as

$$
\begin{gathered}
\left(\begin{array}{cc}
L & 0 \\
0 & L \cos \vartheta
\end{array}\right)\left(\begin{array}{c}
\dot{\vartheta} \\
\dot{\varphi}
\end{array}\right)= \\
\bar{R}_{N K}\left(\begin{array}{ccc}
1 & 0 & -\mathbf{E} \\
0 & 0 & 0
\end{array}\right) R_{N K}^{T} R_{G N}^{T} v_{w}-\bar{R}_{N K}\left(\begin{array}{c}
\mathbf{E} z \\
0
\end{array}\right) \\
\dot{L}=z \quad \dot{z}=u_{z} \\
\dot{\gamma}=k v_{s}\left(\vartheta, \varphi, \mathbf{E}, v_{w}\right) \delta
\end{gathered}
$$

where $\mathbf{E}$ is an aerodynamic parameter of the kite, called glide ratio, and $v_{w}$ is the wind velocity vector in the ground frame. The control inputs of the physical system are the reelout rate $z$ and kite deflection $\delta$ for turning. We design our controller taking $\left(u_{z}, \dot{\gamma}\right)$ as virtual inputs to the kite. We use a proportional controller to get the input $\delta$ that approximately tracks the resulting $\gamma$ demanded by our controller.

\section{Reference Path}

We describe the desired path with its projection on the $y-z$ plane of the ground frame $\{G\}$ and denote it by a smooth curve $\left(Y_{\text {ref }}(\tau), Z_{\text {ref }}(\tau)\right)$ parametrized by a scalar $\tau$. Note that for any tether length there exists a trajectory that, projected in the $y-z$ plane of $\{G\}$, satisfies the desired assignment. Also note that $\tau$ is a parameter controlled by our controller and as a result the speed with which the reference moves along the path is controlled through $\tau$.

The desired path is assumed to have no stationary points, i.e., $\left\|\frac{\partial\left(Y_{\text {ref }}(\tau), Z_{\text {ref }}(\tau)\right)}{\partial \tau}\right\| \neq 0$ for any $\tau \in \mathbb{R}$, or in other words, as $\tau$ changes the point should move along the curve with a non-zero speed. Two such path projections, used in our numerical studies, for a "figure of eight" lemniscate trajectory and an ellipsoidal trajectory are shown in (38),(39).

The length of the tether is controlled specifying the desired velocity of the tether $z_{r e f}(t)$, which is time parameterized unlike the $Y_{r e f}, Z_{r e f}$.

In view of these observations, the output of the system is defined as

$$
y=\left(\begin{array}{ccc}
L & 0 & 0 \\
0 & L \cos \vartheta & 0 \\
0 & 0 & 1
\end{array}\right)\left(\begin{array}{l}
\vartheta \\
\varphi \\
z
\end{array}\right) \quad y_{r e f}=\left(\begin{array}{ccc}
L & 0 & 0 \\
0 & L \cos \vartheta_{r e f} & 0 \\
0 & 0 & 1
\end{array}\right)\left(\begin{array}{l}
\vartheta_{r e f} \\
\varphi_{r e f} \\
z_{r e f}
\end{array}\right)
$$

\section{Controller Design}

\section{A. Error Definition}

This section introduces the error space utilized for the design of the path following controller. Similar to [12], [13], we consider the tracking error vector

$$
\mathbf{e}=R_{N K}^{T}\left(y-y_{r e f}\right)-\epsilon
$$

for a given vector $\epsilon \in \mathbb{R}^{3}$ with non-zero norm. Note that as the norm of the error vector goes to zero, the distance $y-y_{\text {ref }}$ converges to $\|\epsilon\|$, which can be made arbitrarily small.

\section{B. Error dynamics}

Taking the derivative for the error as defined in (3), we get error dynamics in the form,

$$
\dot{\mathbf{e}}=\dot{\gamma} \tilde{S} \mathbf{e}+f(\mathbf{x})+f_{\lambda}(\mathbf{x}) \lambda+g(\mathbf{x}) u+R(\beta) \tilde{S} \epsilon_{o} \dot{\beta}
$$

where $\mathbf{x}=\left(\vartheta, \varphi, \gamma, L, z, \dot{z}_{r e f}, \tau\right)^{T}$ is the system state, $u=(\dot{z}, \dot{\gamma}, \dot{\tau})^{T}$ is the control input and $\lambda=\left(\mathbf{E}, v_{w}, \mathbf{d}\right)^{T}$ is a vector of unknown system parameters. The bilinear dependence on $\mathbf{E} \cdot v_{w}$ in (1) is denoted and estimated as an independent parameter $\mathbf{d}$. The exact expressions for $\tilde{S}, f, f_{\lambda}, g$ are presented in the appendix in (43). We use

$$
\epsilon=R(\beta) \epsilon_{o}
$$

where $\epsilon_{o}$ is a constant vector in $R^{3}$ with non-zero norm and $R(\beta)$ shown in (40) is a rotation matrix with state dependent $\beta$. From the expression for the determinant of $g$ presented in (44) it can be seen that using $\beta=\zeta-\gamma+\pi / 2, g(\mathbf{x})$ is guaranteed to be invertible at all times. $\zeta$ is again a state dependent term defined in (42). Without the state varying $\beta$, $g(\mathbf{x})$ will lose rank for certain states and the system will lose feedback linearizability at those states. For the state varying $\beta$ chosen above, we can show, $\dot{\beta}$ to be of the form,

$$
\dot{\beta}=W(\mathbf{x})^{T} u
$$

where $W(\mathbf{x})$ is a vector in $\mathbb{R}^{3}$. The error dynamics thus take the form,

$$
\dot{\mathbf{e}}=f(\mathbf{x})+f_{\lambda}(\mathbf{x}) \lambda+(g(\mathbf{x})+h(\mathbf{x})) u
$$

where $h(\mathbf{x})=R(\beta) \tilde{S} \epsilon_{o} W(\mathbf{x})^{T}+\left(\begin{array}{lll}\mathbf{0} & \tilde{S} \mathbf{e} & \mathbf{0}\end{array}\right)$.

This brings the error dynamics in the class of systems for which we will prove local convergence of the closed loop system to an ultimate bound in the following theorem, for cases of known and unknown system parameter vector $\lambda$.

\section{Main Result}

We consider systems of the form,

$$
\dot{\mathbf{e}}=f(\mathbf{x})+f_{\lambda}(\mathbf{x}) \lambda+G(\mathbf{x}) u
$$

where $\mathbf{x} \in \mathbb{R}^{n}, u \in \mathbb{R}^{m}$ are the system states and inputs respectively. $\mathbf{e}=l(\mathbf{x}) \in \mathbb{R}^{m}$ is some nonlinear output of the system state. $G(\mathbf{x})$ is possibly non-invertible at certain states, but can be written in the form,

$$
G(\mathbf{x})=g(\mathbf{x})+h(\mathbf{x})
$$

with $g(\mathbf{x})$ always guaranteed to be invertible and $\|g\| \in[a, b]$ for some finite positive constants $a, b$ and $\|h\| \leq \Delta$ for a finite positive constant $\Delta . f, f_{\lambda}, g$ are known functions satisfying $\left\|\frac{d f(\mathbf{x})}{d t}\right\| \leq \Delta_{1}\|\mathbf{e}\|\|u\|,\left\|\frac{d f_{\lambda}(\mathbf{x})}{d t}\right\| \leq \Delta_{2}\|\mathbf{e}\|\|u\|$, $\left\|\frac{d g}{d t}\right\| \leq \Delta_{3}\|\mathbf{e}\|\|u\|$ and $\left\|f_{\lambda}\right\|<\Delta_{4}$ where $\Delta_{1}, \Delta_{2}, \Delta_{3}, \Delta_{4}$ are scalar positive and finite constants.

$\lambda$ is a vector of system parameters in $\mathbb{R}^{p}$. When the parameters $\lambda$ are unknown we use an online estimate of the parameter denoted by $\bar{\lambda}$ and design for an update rule $\dot{\bar{\lambda}}$ assuming $\lambda$ to be unknown constants. We also denote any offline a priori estimates of the parameters by $\hat{\lambda}$. In the 
nominal case when $\lambda$ is known we simply set $\bar{\lambda}=\hat{\lambda}=\lambda$ and $\dot{\bar{\lambda}}=0$ in our adaptive control law.

Note, since $f(\mathbf{x}), f_{\lambda}(\mathbf{x}), g(\mathbf{x}), h(\mathbf{x})$ are functions of only $\mathbf{x}$ we will drop the explicit notation and denote the function evaluated at $\mathbf{x}$ as $f, f_{\lambda}, g, h$. Also we use the following vector operations: $\operatorname{Tanh}(\Theta)$ acting on a vector $\Theta$ represents an element-wise $\tanh (\cdot)$ operating on the elements of $\Theta \cdot \operatorname{Cosh}(\Theta), \operatorname{Sech}(\Theta)$ are diagonal matrices with diagonal entries being the corresponding element-wise operations on elements of vector $\Theta$.

Theorem 1. For a system of the form (8), a control law,

$$
u=q+N \cdot \operatorname{Tanh}(\Theta)
$$

with

$$
\begin{aligned}
\dot{\Theta} & =\operatorname{Cosh}^{2}(\Theta) N^{-1} g^{-1} \nu \\
\nu & =-K_{r} \mathbf{r}-m+\chi \\
\mathbf{r} & =f+f_{\lambda} \bar{\lambda}+g u+K \mathbf{e} \\
m & =\dot{f}+\dot{f}_{\lambda} \bar{\lambda}+\dot{g} u+K h u+f_{\lambda} \dot{\bar{\lambda}} \\
\chi & =-k_{\Theta} g M \operatorname{Tanh}(\Theta)
\end{aligned}
$$

and parameter update law

$$
\dot{\bar{\lambda}}=K_{\lambda} f_{\lambda}^{T}\left(\mathbf{e}+K^{-1} \mathbf{r}\right)-\sigma(\bar{\lambda}-\hat{\lambda})
$$

enforces e, $\mathbf{r}, \Theta, \bar{\lambda}$ to converge to a bounded set tuned using the tuning variables in the control scheme, vi.z., $K, K_{r}, k_{\Theta}, N, K_{\lambda}, \sigma$.

Proof: Consider the error vector defined in (3) with

$$
\dot{\mathbf{e}}=f+f_{\lambda} \lambda+g u+h u .
$$

We would like $f+f_{\lambda} \lambda+g u$ to be close to $-K \mathbf{e}$, for a diagonal positive definite constant matrix $K \succ 0$, with bounded inputs $u$. We thus proceed in a backstepping fashion by defining the backstepping variable

$$
\mathbf{r}=f+f_{\lambda} \bar{\lambda}+g u+K \mathbf{e}
$$

and driving it to zero. Note that, since we only have the estimate $\bar{\lambda}$ of the parameter vector $\lambda$, such variable is defined using the estimate, and later we will design a suitable estimator to compensate for the effect of such discrepancy. Combining (17) with (18) in the nominal case of known parameters, i.e., $\bar{\lambda}=\lambda$, results in

$$
\dot{\mathbf{e}}=-K \mathbf{e}+\mathbf{r}+h u
$$

whereas in the case of unknown parameters we have

$$
\dot{\mathbf{e}}=-K \mathbf{e}+\mathbf{r}+f_{\lambda}(\lambda-\bar{\lambda})+h u .
$$

Differentiating the backstepping variable $\mathbf{r}$ results in

$$
\dot{\mathbf{r}}=\dot{f}+\dot{f}_{\lambda} \bar{\lambda}+\dot{g} u+K \dot{\mathbf{e}}+f_{\lambda} \dot{\bar{\lambda}}+g \dot{u}
$$

where the terms $\dot{f}, \dot{f}_{\lambda}$, and $\dot{g}$ denote the time derivatives of functions $f, f_{\lambda}$, and $g$, respectively. Plugging (20) into (21),

$$
\dot{\mathbf{r}}=m-K^{2} \mathbf{e}+K \mathbf{r}+g \dot{u}+K f_{\lambda}(\lambda-\bar{\lambda})
$$

where the term $m$ is defined as

$$
m=\dot{f}+\dot{f}_{\lambda} \bar{\lambda}+\dot{g} u+K h u+f_{\lambda} \dot{\bar{\lambda}} .
$$

Now considering the Lyapunov function,

$$
\begin{aligned}
V_{1}= & \frac{1}{2}\left(\mathbf{e}^{T} \mathbf{e}+\mathbf{r}^{T} K^{-2} \mathbf{r}\right) \\
\dot{V}_{1}= & \mathbf{e}^{T} \dot{\mathbf{e}}+\mathbf{r}^{T} K^{-2} \dot{\mathbf{r}} \\
= & -\mathbf{e}^{T} K \mathbf{e}+\mathbf{e}^{T} h u+\left(\mathbf{e}^{T}+K^{-1} \mathbf{r}^{T}\right) f_{\lambda}(\lambda-\bar{\lambda}) \\
& +\mathbf{r}^{T} K^{-2}(m+K \mathbf{r}+g \dot{u})
\end{aligned}
$$

In what follows, we proceed by defining a suitable input $\dot{u}$ to enforce the desired decrease of the lyapunov function. Although, the term $e^{T} h u$ in the inequality (24) cannot be cancelled, we design $\dot{u}$ explicitly enforcing a bounded $u$. This can be achieved by defining

$$
u=q+N \cdot \operatorname{Tanh}(\Theta)
$$

with first time derivative

$$
\dot{u}=N \operatorname{Sech}^{2}(\Theta) \dot{\Theta}
$$

where the constants $q$ and $N$ are design parameters and $\Theta$ is an internal state of the controller.

Therefore, designing $\dot{u}$ such that

$$
g \dot{u}=-K_{r} \mathbf{r}-m+\chi,
$$

for some term $\chi$, is equivalent to choosing

$$
\dot{\Theta}=\operatorname{Cosh}^{2}(\Theta) N^{-1} g^{-1}\left(-K_{r} \mathbf{r}-m+\chi\right),
$$

The term $\chi$, in the following, is used to maintain the internal state of the controller $\Theta$ bounded and avoid numerical integration problems.In fact, an unstable internal state $\Theta$ will eventually drive $\operatorname{Sech}(\Theta)$ to $\mathbf{0}$, and the $\dot{\Theta}$ resulting from

$$
g N \operatorname{Sech}^{2}(\Theta) \dot{\Theta}=-K_{r} \mathbf{r}-m+\chi
$$

will be numerically infeasible to integrate. Toward this goal, we update the Lyapunov function introducing an extra term,

$$
V_{2}=V_{1}+\frac{1}{2} k_{\Theta}^{-1} \operatorname{Tanh}^{T}(\Theta) \operatorname{Tanh}(\Theta)
$$

where $k_{\Theta}>0$ is a positive scalar constant. Computing the first time derivative combining with (24) and (26) results in

$$
\begin{aligned}
\dot{V}_{2}= & \dot{V}_{1}+k_{\Theta}^{-1} \operatorname{Tanh}^{T}(\Theta) N^{-1} g^{-1}\left(-K_{r} \mathbf{r}-m+\chi\right) \\
= & -\mathbf{e}^{T} K \mathbf{e}-\mathbf{r}^{T} K^{-2}\left(K_{r}-K\right) \mathbf{r}+\mathbf{e}^{T} h q \\
& +\mathbf{e}^{T} h N \operatorname{Tanh}(\Theta)+\mathbf{r}^{T} K^{-2} \chi \\
& +k_{\Theta}^{-1} \operatorname{Tanh}^{T}(\Theta) N^{-1} g^{-1}\left(-K_{r} \mathbf{r}-m+\chi\right) \\
& +\left(\mathbf{e}^{T}+K^{-1} \mathbf{r}^{T}\right) f_{\lambda}(\lambda-\bar{\lambda}) .
\end{aligned}
$$

Choosing

$$
\chi=-k_{\Theta} g M \operatorname{Tanh}(\Theta) \quad M=\min (N, I)
$$

where $M$ is the element wise minimum of the matrices $N$ and $I$ (the identity matrix), such that,

$$
0<N^{-1} M \leq I \quad 0<M \leq I
$$


results in

$$
\begin{aligned}
\dot{V}_{2}= & -\mathbf{e}^{T} K \mathbf{e}-\mathbf{r}^{T} K^{-2}\left(K_{r}-K\right) \mathbf{r}+\mathbf{e}^{T} h q \\
& +\mathbf{e}^{T} h N \operatorname{Tanh}^{T}(\Theta)-k_{\Theta} \mathbf{r}^{T} K^{-2} g M \operatorname{Tanh}(\Theta) \\
& -k_{\Theta}^{-1} \operatorname{Tanh}^{T}(\Theta) N^{-1} g^{-1} K_{r} \mathbf{r} \\
& -k_{\Theta}^{-1} \operatorname{Tanh}^{T}(\Theta) N^{-1} g^{-1} m \\
& -\operatorname{Tanh}^{T}(\Theta) N^{-1} M \operatorname{Tanh}(\Theta) \\
& +\left(\mathbf{e}^{T}+K^{-1} \mathbf{r}^{T}\right) f_{\lambda}(\lambda-\bar{\lambda}) .
\end{aligned}
$$

Known parameter case. For the case of known parameter $\lambda$ we have $\dot{\bar{\lambda}}=0, \bar{\lambda}=\lambda$ Using the norm inequalities,

$$
\begin{aligned}
\dot{V}_{2} \leq & -\mathbf{e}^{T} K \mathbf{e}-\mathbf{r}^{T} K^{-2}\left(K_{r}-K\right) \mathbf{r} \\
& -\operatorname{Tanh}^{T}(\Theta) N^{-1} M \operatorname{Tanh}(\Theta) \\
& +\|\mathbf{e}\|\|h\|\|q\|+\|\mathbf{e}\|\|h\|\|N\| \\
& +\|\mathbf{r}\|\left\|K^{-2}\right\|\|g\| \\
& +k_{\Theta}^{-1}\left\|N^{-1}\right\|\left\|g^{-1}\right\|\left\|K_{r}\right\|\|\mathbf{r}\| \\
& +k_{\Theta}^{-1}\left\|N^{-1}\right\|\left\|g^{-1}\right\|\|m\|
\end{aligned}
$$

By earlier assumptions on bounds for $\|\dot{f}\|,\left\|\dot{f}_{\lambda}\right\|,\|\dot{g}\|,\|h\|$

$$
\begin{aligned}
\|m\| & \leq\|\dot{f}\|+\left\|\dot{f}_{\lambda}\right\|\|\bar{\lambda}\|+\|\dot{g}\|\|u\|+\|K|\||||\||| u\| \\
& \leq \Delta_{1}\|\mathbf{e}\|+\Delta_{2}\|\mathbf{e}\|\|\bar{\lambda}\|+\Delta_{3}\|\mathbf{e}\|+\|K\| \Delta(30)
\end{aligned}
$$

Implying,

$$
\begin{aligned}
\dot{V}_{2} \leq & -k_{1}\|\mathbf{e}\|^{2}-k_{2}\|\mathbf{r}\|^{2}-k_{3}\|\operatorname{Tanh}(\Theta)\|^{2} \\
& +\|\mathbf{e}\|\left(\Delta(\|q\|+\|N\|)+k_{4}\left(\Delta_{1}+\Delta_{2}\|\bar{\lambda}\|+\Delta_{3}\right)\right) \\
& +\|\mathbf{r}\|\left(k_{1}^{-2}\|g\|+k_{4} k_{6}\right)+k_{4}\|K\| \Delta
\end{aligned}
$$

where

$$
\begin{aligned}
k_{1} & :=\lambda_{\min }(K) \\
k_{2} & :=\lambda_{\min }\left(K^{-2}\left(K_{r}-K\right)\right) \\
k_{3} & :=\lambda_{\min }\left(N^{-1} M\right) \\
k_{4} & :=k_{\Theta}^{-1}\left\|N^{-1}\right\|\left\|g^{-1}\right\| \\
k_{5} & :=\left\|K_{r}\right\|
\end{aligned}
$$

where for a generic matrix $A$, the term $\lambda_{\min }(A)$ denotes the minimum singular value of $A$ and where the constant terms $K, K_{r}, N, k_{\Theta}$, with the restriction $K_{r}>K$, are design parameters introduced earlier. Note that, in the Lyapunov inequality (31), as the terms $e$ and $r$ grow, the quadratic negative terms will eventually dominate the positive linear and bounded terms, resulting in the standard ultimately bounded behaviour of $e$ and $r$. Similar applies to the term $\Theta$, although here, since the $\operatorname{Tanh}(\cdot)$ is not a radially unbounded function, an excessive magnitude of the positive terms might cause $\Theta$ to be unbounded and therefore care should be taken in selection of the design parameters.

Further increasing $k_{1}, k_{\Theta}$ and $k_{2}$ allows us to reduce the ultimate bound on $\mathbf{e}$ and $\mathbf{r}$ axes.

Unknown parameter case. For the case of unknown parameter $\lambda$, we consider the Lyapunov function,

$$
\begin{aligned}
& V_{3}=V_{2}+\frac{1}{2}(\lambda-\bar{\lambda})^{T} K_{\lambda}^{-1}(\lambda-\bar{\lambda}) \\
& \dot{V}_{3}=\dot{V}_{2}-(\lambda-\bar{\lambda})^{T} K_{\lambda}^{-1} \dot{\bar{\lambda}}
\end{aligned}
$$

Using (28),

$$
\begin{aligned}
\dot{V}_{3}= & -\mathbf{e}^{T} K \mathbf{e}-\mathbf{r}^{T} K^{-2}\left(K_{r}-K\right) \mathbf{r}+\mathbf{e}^{T} h q \\
& +\mathbf{e}^{T} h N \operatorname{Tanh}(\Theta)-k_{\Theta} \mathbf{r}^{T} K^{-2} g M \operatorname{Tanh}(\Theta) \\
& -k_{\Theta}^{-1} \operatorname{Tanh}^{T}(\Theta) N^{-1} g^{-1} K_{r} \mathbf{r} \\
& -k_{\Theta}^{-1} \operatorname{Tanh}^{T}(\Theta) N^{-1} g^{-1} m \\
& -\operatorname{Tanh}^{T}(\Theta) N^{-1} M \operatorname{Tanh}(\Theta) \\
& +(\lambda-\bar{\lambda})^{T}\left(f_{\lambda}^{T}\left(\mathbf{e}+K^{-1} \mathbf{r}\right)-K_{\lambda}^{-1} \dot{\bar{\lambda}}\right) .
\end{aligned}
$$

Choosing the parameter update law,

$$
\dot{\bar{\lambda}}=K_{\lambda} f_{\lambda}^{T}\left(\mathbf{e}+K^{-1} \mathbf{r}\right)-\sigma(\bar{\lambda}-\hat{\lambda})
$$

where $K_{\lambda}$ and $\sigma$ are positive definite, diagonal matrices, we obtain

$$
\begin{aligned}
\dot{V}_{3}= & -\mathbf{e}^{T} K \mathbf{e}-\mathbf{r}^{T} K^{-2}\left(K_{r}-K\right) \mathbf{r}+\mathbf{e}^{T} h q \\
& +\mathbf{e}^{T} h N \operatorname{Tanh}(\Theta)-k_{\Theta} \mathbf{r}^{T} K^{-2} g M \operatorname{Tanh}(\Theta) \\
& -k_{\Theta}^{-1} \operatorname{Tanh}^{T}(\Theta) N^{-1} g^{-1} K_{r} \mathbf{r} \\
& -k_{\Theta}^{-1} \operatorname{Tanh}^{T}(\Theta) N^{-1} g^{-1} m \\
& -\operatorname{Tanh}^{T}(\Theta) N^{-1} M \operatorname{Tanh}(\Theta) \\
& -\sigma(\lambda-\bar{\lambda})^{T}(\bar{\lambda}-\hat{\lambda}) .
\end{aligned}
$$

The last term in $\dot{V}_{3}=-\sigma(\lambda-\bar{\lambda})^{T}(\bar{\lambda}-\hat{\lambda})$ is always negative definite outside a box in $\mathbb{R}^{p}$ defined by the values of $\lambda$ and $\hat{\lambda}$ and this keeps the estimates $\bar{\lambda}$, bounded. The norm of $m$ will now have the bound

$$
\begin{aligned}
\|m\| \leq & \|\dot{f}\|+\left\|\dot{f}_{\lambda}\right\|\|\bar{\lambda}\|+\|\dot{g}\|\|u\|+\|K\|\|h\|\|u\| \\
& +\left.\left\|f_{\lambda}\right\|\right|^{2}\left\|K_{\lambda}\right\|\left(\|\mathbf{e}\|+\left\|K^{-1}\right\|\|\mathbf{r}\|\right)+\|\sigma\|\|\lambda-\hat{\lambda}\| \\
\leq & \Delta_{1}\|\mathbf{e}\|+\Delta_{2}\|\mathbf{e}\|\|\bar{\lambda}\|+\Delta_{3}\|\mathbf{e}\|+\|K\| \Delta \\
& +\Delta_{4}^{2}\left\|K_{\lambda}\right\|\|\mathbf{| e}\|+\Delta_{4}^{2}\left\|K_{\lambda}\right\|\left\|K^{-1}\right\|\|\mathbf{r}\| \\
& +\|\sigma\|\|\lambda-\hat{\lambda}\|
\end{aligned}
$$

Since the $\|m\|$ is still bounded linearly in terms of $\|\mathbf{e}\|,\|\mathbf{r}\|$, $\dot{V}_{3}$ also takes the same form as (31) with a added constant $\|\sigma\|\|\lambda-\hat{\lambda}\|$. Thus with some reasonable a priori estimate of parameters $\hat{\lambda}$ such that $\|\lambda-\hat{\lambda}\|$ is bounded, the controller will converge to a bounded ellipsoid in the $(\mathbf{e}, \mathbf{r}, \Theta, \bar{\lambda})$ space. The boundary of the ellipsoid satisfies the equation $\dot{V}_{3}=0\left(\dot{V}_{2}=0\right.$, when $\lambda$ is known). As long as for the chosen tuning variables and apriori estimate $\hat{\lambda}$, the ellipsoid boundary satisfies the strict inequality $\|\operatorname{Tanh}(\Theta)\|_{\infty}<1$, the convergence of states $(\mathbf{e}, \mathbf{r}, \Theta, \bar{\lambda})$ to the ellipsoid is guaranteed.

\section{NUMERICAL RESUlTS}

We test the control scheme described in Theorem 1 for our kite system, with the path following error system dynamics as described in (4) under constant but unknown wind vector $v_{w}=(10,0,0)$, constant unknown glide ratio $\mathbf{E}=5$ with a a priori guesses $\hat{v}_{w}=(9,0,0), \hat{\mathbf{E}}=6, \hat{\mathbf{d}}=(45,0,0)$.

The controller tuning parameters were chosen as, $\epsilon_{o}=(-0.1,-0.1,0)^{T}, K=\operatorname{diag}(4,4,10), K_{r}=$ $\operatorname{diag}(20,20,20), \quad k_{\Theta}=10, \sigma=0.1, K_{\lambda}=$ $\operatorname{diag}(0.5,0.5,0.5,0.5,0.5,0.01,0.01), q=(0,0,0), N=$ 
$\operatorname{diag}(2,50,20)$, where $\operatorname{diag}(x)$ represents a diagonal matrix with diagonal entries given by $x$.

The kite is initialized at an initial condition close to the ground, to show the behaviour of the controller for a large starting error and a long transient phase. The reel-out reference rate is set to be $0.5 \mathrm{~m} / \mathrm{s}$ during traction and at -1 $\mathrm{m} / \mathrm{s}$ during the reel-in phase. The reference path sizes are different during the two phases and we switch the reference paths when the phases are switched. The kite is set to be in the traction initially. When the tether length exceeds 50 meters, we switch to retraction mode and reel-in till the tether length becomes less than 35 meters, at which point we switch back to the traction phase, completing a full pumping cycle.

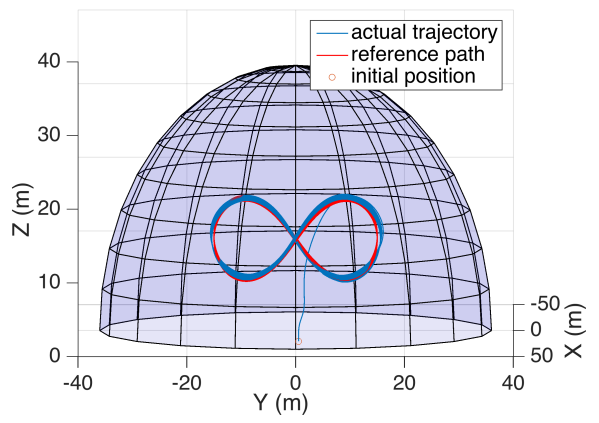

Fig. 2: Path following of lemniscate figures in traction phase

Figures 2,3,4 show path tracking for different reference paths in the traction and retraction phase. In the paths tested the controller shows fast convergence of the errors to a small bound with good tracking performance.

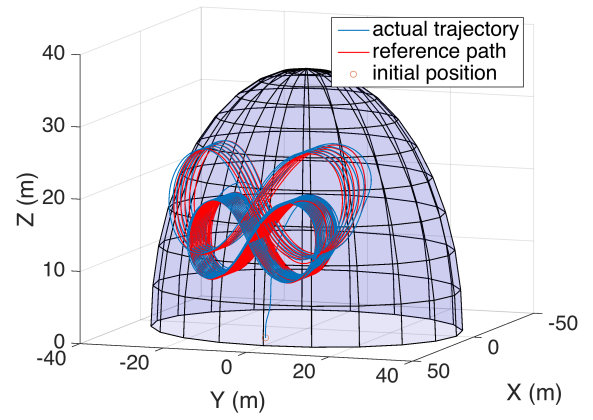

Fig. 3: Path following of lemniscate in full pumping cycle

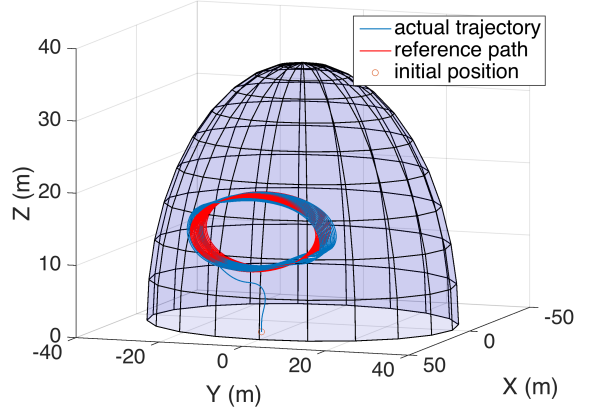

Fig. 4: Path following of ellipsoidal orbits in traction phase

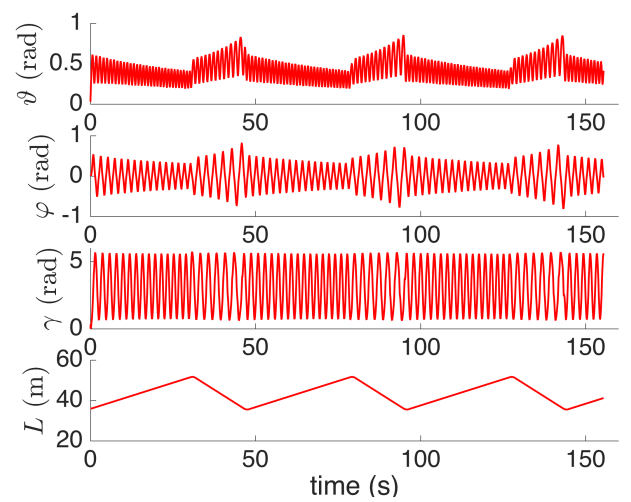

Fig. 5: Kite state evolution through the pumping cycle

Figure 5 shows the evolution of the states for the kite as it flies figures of eight during several pumping cycles. The tether length tracks the different reference slopes during the cycle and maintains tracking of the kite reference position. The minimum elevation angle $\vartheta$ decreases as the tether length increase and vice versa. This occurs because we have demanded a reference path with a constant minimum height characteristic which is desirable to higher power generation. The virtual control inputs given by the controller $u=$

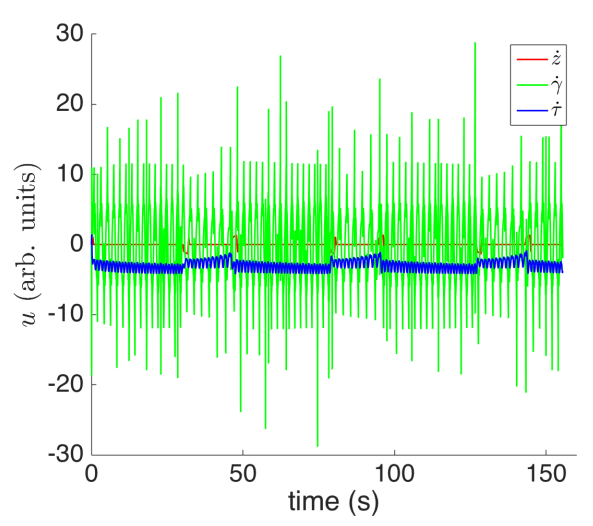

Fig. 6: Control inputs through the pumping cycle

$(\dot{z}, \dot{\gamma}, \dot{\tau})$ are shown in Fig.6. None of the virtual controllers become saturated at any time as we had allowed for a maximum of amplitude of 2,50,20 for $\dot{z}, \dot{\gamma}, \dot{\tau}$, respectively. Thus the internal states of the controller $\Theta$ also remain bounded (Fig.7). The estimates for the parameters show bounded values as well. Note that the controller only guaranteed closed loop stability of the system and does not require or guarantee the convergence of the estimates to their true values. This is seen in Fig. 8

\section{CONCLUSiON}

This paper presents an adaptive path following controller for kite systems with parameter mismatch and with unknown wind velocity vector. Under mild assumptions, the controller steers kite to a tube centered around a predefined geometric path. The tube diameter is determined by the choice of the design parameters of the controller. The effectiveness of the 


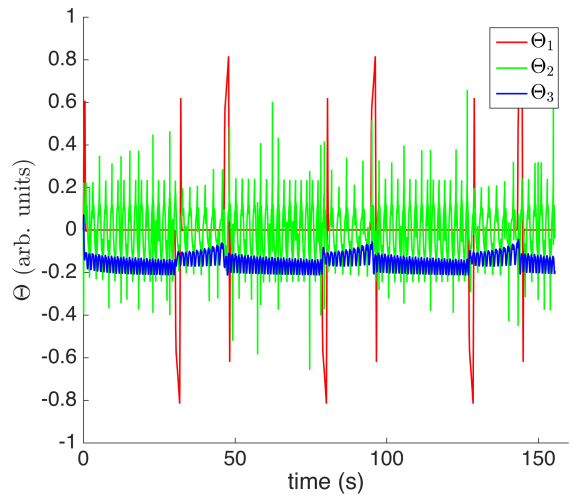

Fig. 7: Internal states of the controller $\Theta$

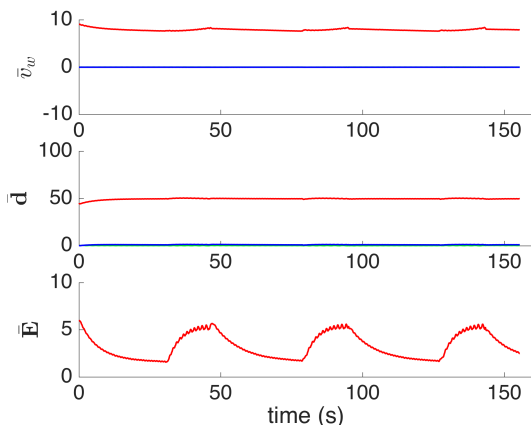

Fig. 8: Parameter estimates $\bar{\lambda}=\left(\bar{v}_{w}, \overline{\mathbf{d}}, \overline{\mathbf{E}}\right)$. Vector components of $\bar{v}_{w}, \overline{\mathbf{d}}$ in red, blue and green colors.

proposed strategy is demonstrated via numerical results on multiple geometric desired paths and pumping cycle flights.

\section{APPENDIX}

The figure of eight trajectory as tracked in figures 2,3 has the following projection on the $y-z$ plane,

$$
Y_{\text {ref }}=\frac{a \cos \tau}{1+\sin ^{2} \tau} \quad Z_{\text {ref }}=h+\frac{a \sin \tau \cos \tau}{1+\sin ^{2} \tau}
$$

with $a$ being the width of the lemniscate and $h$ the height of the center of the lemniscate. We use the values for $(a, h)=$ $(15,15)$ during the reel out phase and use a larger figure with $(a, h)=(20,20)$ during the reel in phase to have a different retraction path.

The ellipsoidal trajectory as tracked in 4 has the following projection on the $y-z$ plane,

$$
Y_{\text {ref }}=a \cos \tau \quad Z_{\text {ref }}=h+\frac{a}{e} \sin \tau
$$

with $a$ being the width of the major axis for the ellipse, $e$ being its eccentricity and $h$ the height of its center.

For the reference reel-out rate $z_{r e f}(t)$ we use a constant positive reel out rate $c_{o}=0.5$ and a constant reel-in rate $c_{o}=-1$. The reference length $L_{r e f}(t)$ can then be written as, $L_{r e f}(t)=L_{r e f}(0)+c_{o} \cdot t$ where $L_{r e f}(0)$ is the initial tether length of the kite.

$$
R(\beta)=\left(\begin{array}{ccc}
\cos \beta & -\sin \beta & 0 \\
\sin \beta & \cos \beta & 0 \\
0 & 0 & 1
\end{array}\right), \quad \tilde{S}=\left(\begin{array}{ccc}
0 & 1 & 0 \\
-1 & 0 & 0 \\
0 & 0 & 0
\end{array}\right)
$$

$$
\begin{aligned}
T= & \left\|\left(\begin{array}{c}
\sin \vartheta_{r e f} \varphi_{r e f} \partial_{\tau} \vartheta_{r e f}-\cos \vartheta_{r e f} \partial_{\tau} \varphi_{r e f} \\
\partial_{\tau} \vartheta_{r e f}
\end{array}\right)\right\| \\
\zeta= & \angle\left(\begin{array}{c}
\sin \vartheta_{r e f} \varphi_{r e f} \partial_{\tau} \vartheta_{r e f}-\cos \vartheta_{r e f} \partial_{\tau} \varphi_{r e f} \\
\partial_{\tau} \vartheta_{r e f}
\end{array}\right)(42) \\
\dot{\mathbf{e}}= & R_{N K}^{T}\left(\begin{array}{ccc}
0 & -L T \sin (\zeta) \\
0 & R_{N K} \tilde{S} \epsilon & L T \cos (\zeta) \\
1 & 0
\end{array}\right)\left(\begin{array}{c}
\dot{z} \\
\dot{\gamma} \\
\dot{\tau}
\end{array}\right) \\
& +\dot{\gamma} \tilde{S} \mathbf{e}+R(\beta) \tilde{S} \dot{\beta} \epsilon_{O} \\
& -R_{N K}^{T}\left(\begin{array}{ccc}
L & 0 & 0 \\
-L \sin \vartheta_{r e f} \varphi_{r e f} & L \cos \vartheta_{r e f} & 0 \\
0 & 0 & 1
\end{array}\right)\left(\begin{array}{c}
\partial_{t} \vartheta_{r e f} \\
\partial_{t} \varphi_{r e f} \\
\dot{L}_{r e f}
\end{array}\right) \\
& +R_{N K}^{T}\left(\begin{array}{ccc}
\cos \vartheta \varphi-\cos \vartheta_{r e f} \varphi_{r e f}+\mathbf{E} \sin \vartheta \varphi \cos \gamma-\mathbf{E} \sin \gamma \\
0
\end{array}\right) z \\
& +R_{N K}^{T}\left(\begin{array}{ccc}
1 & 0 & 0 \\
-\sin \vartheta \varphi & 1 & 0 \\
0 & 1
\end{array}\right) R_{N K}\left(\begin{array}{ccc}
1 & 0 & -\mathbf{E} \\
0 & 0 & 0 \\
0 & 0 & 0
\end{array}\right) R_{N K}^{T} R_{G N}^{T} v_{w} \\
= & \dot{\gamma} \tilde{S} \mathbf{e}+R(\beta) \tilde{S} \dot{\beta} \epsilon_{o}+f+f_{\lambda} \lambda+g u \\
\operatorname{det}(g) & =L T \epsilon_{o_{1}}
\end{aligned}
$$

\section{REFERENCES}

[1] R. Luchsinger, "Pumping cycle kite power," in Airborne Wind Energy, ser. Green Energy and Technology, U. Ahrens, M. Diehl, and R. Schmehl, Eds. Springer Berlin Heidelberg, 2013, pp. 47-64.

[2] M. L. Loyd, "Crosswind kite power," Journal of Energy, vol. 4, pp. 106-111, Jun. 1980.

[3] B. Houska and M. Diehl, "Optimal control for power generating kites," in In Proceedings of the 9th European Control Conference, Kos, Greece, 2007, p. 35603567.

[4] — , "Robustness and stability optimization of power generating kite systems in a periodic pumping mode," in Control Applications (CCA), 2010 IEEE International Conference on, Sept 2010, pp. 2172-2177.

[5] M. Erhard, G. Horn, and M. Diehl, "A quaternion-based model for optimal control of the SkySails airborne wind energy system," ArXiv e-prints, Aug. 2015.

[6] J. H. Baayen and W. J. Ockels, "Tracking control with adaption of kites," ArXiv e-prints, Nov. 2010.

[7] M. Erhard and H. Strauch, "Control of towing kites for seagoing vessels," IEEE Trans. Control Syst. Technol., vol. 21, pp. 1629-1640, Nov. 2012.

[8] M. Erhard and H. Strauch, "Flight control of tethered kites in autonomous pumping cycles for airborne wind energy," ArXiv e-prints, Sep. 2014.

[9] L. Fagiano, A. Zgraggen, M. Morari, and M. Khammash, "Automatic crosswind flight of tethered wings for airborne wind energy: Modeling, control design, and experimental results," Control Systems Technology, IEEE Transactions on, vol. 22, no. 4, pp. 1433-1447, July 2014.

[10] A. Ilzhfer, B. Houska, and M. Diehl, "Nonlinear mpc of kites under varying wind conditions for a new class of large-scale wind power generators," International Journal of Robust and Nonlinear Control, vol. 17, no. 17, pp. 1590-1599, 2007. [Online]. Available: http://dx.doi.org/10.1002/rnc. 1210

[11] S. Gros, M. Zanon, and M. Diehl, "Control of airborne wind energy systems based on nonlinear model predictive control amp; moving horizon estimation," in Control Conference (ECC), 2013 European, July 2013, pp. 1017-1022.

[12] A. Alessandretti, A. Aguiar, and C. Jones, "Trajectory-tracking and path-following controllers for constrained underactuated vehicles using model predictive control," in Control Conference (ECC), 2013 European, July 2013, pp. 1371-1376.

[13] R. Carona, A. P. Aguiar, and J. Gaspar, "Control of unicycle type robots tracking, path following and point stabilization." 